\title{
Technical Integration of Defense Threat Reduction Agency (DTRA) Location Related Funded Projects into the DOE Knowledge Base
}

C.A. Schultz, J. Bhattacharyya, M.P. Flanagan, P. Goldstein, S.C. Myers and J. Swenson

March 14, 2000

Lawrence

Livermore

National

Laboratory 


\section{DISCLAIMER}

This document was prepared as an account of work sponsored by an agency of the United States Government. Neither the United States Government nor the University of California nor any of their employees, makes any warranty, express or implied, or assumes any legal liability or responsibility for the accuracy, completeness, or usefulness of any information, apparatus, product, or process disclosed, or represents that its use would not infringe privately owned rights. Reference herein to any specific commercial product, process, or service by trade name, trademark, manufacturer, or otherwise, does not necessarily constitute or imply its endorsement, recommendation, or favoring by the United States Government or the University of California. The views and opinions of authors expressed herein do not necessarily state or reflect those of the United States Government or the University of California, and shall not be used for advertising or product endorsement purposes.

Work performed under the auspices of the U. S. Department of Energy by the University of California Lawrence Livermore National Laboratory under Contract W-7405-Eng-48.

This report has been reproduced

directly from the best available copy.

Available to DOE and DOE contractors from the

Office of Scientific and Technical Information

P.O. Box 62, Oak Ridge, TN 37831

Prices available from (423) 576-8401

http:/ / apollo.osti.gov/bridge/

Available to the public from the National Technical Information Service

U.S. Department of Commerce

5285 Port Royal Rd.,

Springfield, VA 22161

http://www.ntis.gov/

OR

Lawrence Livermore National Laboratory

Technical Information Department's Digital Library

http://www.llnl.gov/tid/Library.html 


\section{Technical Integration of Defense Threat Reduction Agency (DTRA) Location Related Funded Projects into the DOE Knowledge Base}

Craig A. Schultz, Joydeep Bhattacharyya, Megan P. Flanagan, Peter Goldstein, Stephen

C. Myers, Jennifer Swenson

Location Project, Ground-Based Nuclear Explosion Monitoring (GNEM)

Lawrence Livermore National Laboratory

\section{Summary}

This document directly reviews the current Defense Threat Reduction Agency (DTRA) PRDA contracts and describes how they can best be integrated with the DOE CTBT R\&D Knowledge Base. Contract descriptions and numbers listed below are based on the DOE CTBT R\&D Web Site - http://www.ctbt.rnd.doe.gov. More detailed information on the nature of each contract can be found through this web site. In general, the location related PRDA contracts provide products over a set of categories. These categories can be divided into five areas, namely

- Contextual map information

- Reference event data

- Velocity models

- Phase detection/picking algorithms

- Location techniques

Therefore, for this document we focus on only these limited portions of the DOE KB delivery.

On a case-by-case basis we provide an overview of the products generated by each external DTRA PRDA contract and we describe in detail the feasibility of integrating these products with the DOE CTBT R\&D KB. When it is feasible, we provide a detailed plan for how the product can best be evaluated and then, if appropriate, merged with the current methods and data in the DOE KB. For convenience, Section I provides a concise table summarizing the potential value added by each of these external products. The summary table describes what component of the KB will be most impacted. Section II provides a more detailed review of the research proposed in each PRDA contract, the work accomplished by the project's Principal Investigators (PIs), and the relevance to the DOE KB. We then elaborate on the most effective method of integration.

Details in this report are based on many sources of information. However, we rely most heavily on the research results presented at each of the meetings of the Seismic Research Symposium: Technologies for Monitoring the Comprehensive Nuclear-Test Ban Treaty; and, whenever possible we base the results on the direct interaction with the project's PIs. Note that this product information is most effectively integrated into the DOE KB when the PIs provide us direct access to the raw data and the technical algorithms used to generate their results. These rather rare instances are noted in the detailed text. In the majority of cases the products have not been directly provided to LLNL. Unfortunately, 
the results of these less accessible products will take more time and effort to integrate and only a limited integration likely will be possible. 
Section I: Summarv Review

Contract Number

DSWA 01-97-0006

Integration Summary

DTRA 01-99-C-0025

Contextual integration: merge tectonic and velocity maps, QC, evaluate consistency with other contextual information

DSWA 01-97-C-0124 Model integration: merge teleseismic based velocity models with our MENA model, QC, evaluate predictability against our models, adapt merged model, then evaluate improvement in location.

DSWA 01-98-C-0155 Algorithm integration: compare performance of automated picking algorithms with other algorithms, determine level of improvement, adopt if it outperforms current techniques.

DSWA 01-98-C-0159 Algorithm integration: use results to develop a guideline for implementing detectors globally, determine improvement gained by using guidelines versus the more standard approaches.

DTRA 01-99-P-0015 Algorithm integration: compare the Gaussian beam raytracing technique with current tau-p approach, technique with better performance (accuracy and efficiency) can be implemented.

DSWA 01-98-C-0159 Algorithm integration: compare performance of this automated detection algorithm with other algorithms, determine level of improvement, adopt if it outperforms current techniques.

DSWA internal

Algorithm integration: assess the location technique. Assuming uncertainty can be predicted, compare with other location algorithms and integrate where it outperforms other techniques.

DSWA 01-97-C-0127 Algorithm integration: currently being integrated through an internal contract designed to build additional capabilities on a grid search code to enhance location and uncertainty estimates.

DSWA 01-97-C-151 Algorithm integration: compare performance of automated picking algorithms with other algorithms, determine level of improvement, adopt if it outperforms current techniques.

DSWA 01-97-C-0016 Algorithm integration: evaluate performance of beam estimation program against other $\mathrm{KB}$ approaches, quantify any improvement, adopt best performing technique.

DSWA 01-98-C-0032 Model integration: merge regionalized velocity models with our MENA model, QC, evaluate predictability against our models, adapt merged model, then evaluate improvement in location.

DSWA 01-98-1-0005 Algorithm integration: assess the location technique. Compare with other relative (e.g. JHD) location algorithms and integrate where it outperforms other techniques.

DSWA01-99-C-0019 Model integration: merge teleseismic based velocity models with our MENA model, QC, evaluate predictability \& improvement in location.

DWSA 01-97-C-151 Data and Model integration: integrate GT0 quarry blasts in Israel, revise velocity models based on delivered models, evaluate predictability \& location improvement 



\section{Section II: Detailed Reviews}

\section{DSWA 01-97-0006}

Regional Travel Time and Amplitude Ratio ....

By Eric Sandvol et al., Cornell University

The primary deliverable from this contract is the Middle East and North Africa GIS developed at Cornell University. The coverage maps have already been incorporated into the MENA database which is being developed at the Livermore Laboratory. The coverage maps in ArcInfo format have been delivered and integrated with LLNL's database. To better use these maps in our seismic analysis, we will work to collaborate with the Cornell group to evaluate the integration of the "Profile Maker" program and other tools to query velocity models. To compute correction surfaces using the MENA GIS system, the Cornell group has demonstrated a finite difference technique for predicting travel-times. This is one of the focus areas of the Livermore group and we expect to benchmark and enhance the capabilities of our method by integrating it with Cornell's technique.

\section{DTRA01-99-C-0025}

Source-Specific Station Corrections for Regional Phases at International Monitoring System Stations

By Xiaoping Yang, Keith McLaughlin, Robert North

Center for Monitoring Research (CMR), SAIC

The goal of this CMR project was to improve seismic event location by developing Source Specific Station Corrections for IMS stations. Many of the techniques used in this study are redundant and provide less resolution than those being used by the Location Group at LLNL. We are currently analyzing the contents of the CMR database. Those portions found to be complementary and supplementary to the DOE KB will be integrated. For example, we have found the CMR Reviewed Event Bulletin (REB) extremely valuable and are exploring means by which we might first obtain the full CSS3.0 schema for the REBs, and then integrate these bulletins into the DOE KB. An additional step in the integration of the REBs is to characterize the CMR event waveform picks, comparing their variance with the variance of our own waveform picks

\section{DSWA 01-97-C-0124}

Utilizing three-dimensional seismic velocity models in regional and teleseismic event location

By Michael Antolik, Goran Ekstrom, Adam Dziewonski, Lapo Boschi, Ivan Kitov

The investigators demonstrate that $3 \mathrm{D}$ velocity models can significantly improve teleseismic location accuracy over 1D models. They demonstrate the effectiveness of this technique by improving locations by 20 to $30 \mathrm{~km}$. We can easily integrate this work into our efforts to locate events at regional distances. While the Harvard group has focused 
on teleseismic distances, their technique is similar to our regionalization efforts. We have done some integration of this work already by using the RUM which are made from the same dataset of global $\mathrm{P}$ wave travel timepicks and relocated events as the Harvard $3 \mathrm{D}$ velocity model. We have access to their model and can test it (as well as other 3D models) in the same way we have implemented our MENA model. 3d tomography models of high resolution can be combined with our 1 by 1 degree crust and sediment velocity models to construct and test complete $3 \mathrm{D}$ models to improve locations at regional distances.

\section{DSWA 01-98-C-0155}

Automatic interpretation of regional seismic signals using the CUMSUM-SA algorithms. By Der, McGarvey, and Schumway CTBT99 paper

Changes in the properties of the cumulative sum of a seismogram are used to pick arrivaltime onsets. Change in the variance of the CUMSUM is detected by finding the time that maximizes the likelihood that a change in the seismogram statistics occurred. The significance of the change is measured with an F-statistic, and only those arrivals passing the F-test are kept. A simulated annealing (SA) method for finding onsets is also explored, although the F-statistic-approach outperforms SA. It is noted that pre-filtering (pre-whitening) is an important aspect of the processing. It should also be noted that the tests are not performed on continuous data. Event windows are used. There may be some circularity in the tests since the sensitivity of the CUMSUM method is peaked in the center of the window. The results of this study are encouraging, but the relative merits of the CUMSUM technique and other automatic picking methods are not explored in the paper. The integration effort for this contract focuses on method evaluation. If the CUMSUM method is found to be superior to other methods, then it should be adopted.

\section{DSWA 01-98-C-0159}

Simulating seismic signal detectors

by Federenko and Husebye

This contract looks at methods for improving the detection of seismic signals at a global set of stations. The commonly used STA/LTA detector is compared with the Kolmogorov-Smirnov (K-S) detector. Signals are passed through the detectors and various combinations of the station components are combined with differing levels of synthetically generated noise. The results show that STA/LTA responds more poorly to noise spikes in the data. The K-S technique was robust with respect to these spikes. The $\mathrm{K}-\mathrm{S}$ technique performs well on flat signals, but not surprisingly, performs poorly on sharp signals. The investigators effectively identify the strengths and limitations of each technique. This information will be integrated into the Knowledge Base as a guideline to decide which automated pickers will be utilized in which regions based on specific signal and noise characteristics.

\section{DTRA01-99-P-0015}

Seismic Event Location in 3-D Velocity Models Using Initial and Secondary Arrival Times Computed with Dynamic Ray Tracing 
By Ivan Henson, Xiaoxi Ni, and Wilmer Rivers

Multimax, Inc.

The goal of this project is to add an option to the existing LocSAT location software (Center for Monitoring Research) that will facilitate the use of travel times and travel time derivatives computed by Gaussian beam ray tracing in seismic event location. In addition, the graphical interface that was developed for the 2-D beam software will be extended to display the input 3-D models and display the raypaths that will make up the beams. Computation speed, flexibility, and the extension from 2 to 3 dimensions are several of the advantages that this technique has over existing methods of computing travel times. The 3-D Gaussian beam ray-tracing capability of this software is innovative and should be tested and evaluated with the Knowledge Base. We recommend obtaining a copy of the latest version of the software, and testing it against similar ray tracers currently in use at LLNL

\section{DSWA 01-98-C-0159}

First Breaks- Automatic Pickings of P- and S- Onsets in Seismic Regions By Husebye and Federenko

This paper describes the development of an automated seismic picking algorithm that may be suitable for the ongoing research at LLNL. Though this algorithm is still a prototype we will compare this technique to the other automated picking techniques we are using on continuous data in MENA and, if it improves the picking, we can integrate it into our seismic analysis. This technique would likely be most beneficial in the analysis of local network data and may be most useful for the LLNL deployments in the Middle East. In the future, we plan to test this technique using calibration datasets available in LLNL.

\section{DSWA internal}

A comparative study of two azimuth-based non-standard location methods By R.S. Jih

Jih has developed an alternative technique for locating events in uncalibrated or poorly calibrated areas. Further studies that assess its variability from region to region and provide errors in the estimated location are needed before we would recommend integrating this technique with the knowledge base.

\section{DSWA 01-97-C-0127}

Improved focal depth determination for use in CTBT monitoring

By William Rodi MIT

Murphy et al. Maxwell Techologies

This work addresses the use of later arriving phases to help constrain the focal depth of a seismic event as well as developing a new algorithm for computing formal confidence intervals associated with an event's location. We currently have a small contract with Bill 
Rodi at MIT to integrate this software into our Knowledge Base (KB) because it is shown to be a robust technique. As part of this integration, we hope to continue this collaboration to take advantage of newly acquired data provided by the IMS. The new IMS stations will each be arrays of instruments which will allow both azimuth and slowness measurements of arriving phases to be made. Our current efforts are to include this additional information into the location algorithm to provide further constraints on event locations and better defined, more reliable uncertainty estimates. In addition, this technique can be extended to be consistent with the KB and may eventually serve as the template for the next generation location code (after EVLOC).

\section{DSWA 01-97-C-0151}

Robust procedures for automatic precise location of regional seismic events By Pinsky, P., A. Shapira

A multi-stage process for automated phase picking and location is presented. First, an envelope technique is used to make a first cut at picks and a location. Second, an automated phase arrival technique (similar to Husebye and Fedorenko, CTBT 99 abstracts) refines the phase picks. A robust grid search method is used to determine the final location.

Many components of this paper are currently being evaluated for possible integration. The automatic phase picking algorithm should be examined. LLNL is integrating similar robust grid-search location algorithms; however, performance of each technique should be evaluated. LLNL has integrated the much of the Israeli GT database that is referenced in this paper, and LLNL has an on-going coordination effort to integrate GT data in Israel.

\section{DSWA 01-97-0016}

Accuracy and uncertainty in single-station event location By Jay Pulliam, Cliff Frohlich, and R. Brian Schlottmann

The authors develop a promising technique for improving bearing estimation on 3component stations for seismic events in the 3-4.5 magnitude range. They first show that $1 \mathrm{D}$ models of earth structure based on receiver functions can be used to predict waveforms in regions with simple structures. Using these models, bearings are estimated over the entire $\mathrm{P}$ and $\mathrm{P}-\mathrm{to}-\mathrm{Sv}$ converted energy windows. Accounting for polarization of the $\mathrm{P}$ and SV energy in the waveform, the direction of the event is estimated from a moving 3-D covariance model operating in the phase window. Given the promising results, this technique may prove useful in optimizing our bearing estimates using 3component stations. This technique will be compared with other 3-component estimators in the knowledge base and improvement will be evaluated. The best performing technique will be incorporated. 


\section{DSWA 01-98-C-0032}

Development of 3-D Crustal and Upper Mantle Velocity Models of Northern Eurasia and North America to Refine Location of Regional Seismic Events

By Ryaboy

Russian Federation/United States Calibration Working Group

Center for Monitoring Research

The Russian Federation/CMR working group is in the process of developing and evaluating 3-D velocity models of Northern Eurasia. This line of research is similar and therefore of interest to the Location group at LLNL. At the time the abstract was written, 5 crustal and upper mantle models of Northern Eurasia had been tested. We recommend testing their final and best Northern Eurasia model against our own, and evaluate the uncertainty in both models.

\section{DSWA 01-98-1-0005}

Relocation of Global Events Using the L1-Norm and Source Specific Station Terms By Peter Shearer

This paper describes a seismic event relocation technique. The author computes seismic station specific calibration surfaces that are used in the location algorithm. This approach is similar to that adopted by the event location research group at LLNL. We will analyze the calibration surfaces as computed by the author and will attempt to incorporate them in our location algorithm.

\section{DSWA01-99-C-0019}

Construction of a 3-D P and S model of the crust and upper mantle to improve regional locations in W. China, Central Asia, and parts of the Middle East, By M. Ritzwoller et al

Ritzwoller et al are developing 3-D velocity models for W. China, Central Asia, and parts of the Middle East for use in developing correction surfaces for improved regional location. We have been integrating parts of this work through a number of mechanisms including a surface wave workshop and informal collaborations. Integration of current and future models will require evaluation of uncertainties in the input data and resulting models using ground truth such as recordings of PNEs.

\section{DWSA-A01-97-C-151}

Source characterization of ground truth quarry blasts in Israel and calibration of local stations

By Y. Gitterman and A. Shapira

Four mining explosions recorded by the Israel Seismic Network constitute calibration shots with Ground Truth category GT0 in the Middle East. Several aspects of the seismic data are quantified (travel times, spectral modulation, waveform correlation, 